\title{
Quarkonium and heavy flavour production measurements at ATLAS
}

\author{
Stefanos Leontsinis* \\ on behalf of the ATLAS Collaboration \\ National Technical University of Athens, Brookhaven National Laboratory \\ E-mail: stefanos.leontsinis@cern.ch
}

\begin{abstract}
A large number of detailed measurements of various charm states production $\left(J / \psi, \psi(2 S), \chi_{c}\right)$ at the ATLAS experiment at the LHC at centre-of-mass energies of $2.76 \mathrm{TeV}, 7 \mathrm{TeV}, 8 \mathrm{TeV}$, and $13 \mathrm{TeV}$ is presented. These measurements extend in reach and precision beyond those currently available, and are compared to a variety of the latest theoretical predictions. Measurements of the associated production of quarkonium with a vector boson using the ATLAS Run-1 dataset are also presented. These rare processes provide new insight into QCD models of quarkonium production, but also provide new opportunities to study double parton scattering, including crosssection measurements in single and double parton scattering dominated regimes and a precise assessment of the $\sigma_{\text {eff }}$ parameter governing the effective spatial area of parton-parton interactions at a variety of energy scales. Finally, latest results from the ATLAS experiment on heavy flavour production and decay properties at ATLAS are additionally presented. These results include determination of the decay properties of the $\Lambda_{b}$ baryon and new measurements of $B_{c}^{+}$production cross-sections and branching ratio measurements of the $B_{c}^{+} \rightarrow J / \psi D^{*+}$ decay.
\end{abstract}

The European Physical Society Conference on High Energy Physics

22-29 July 2015

Vienna, Austria

${ }^{*}$ Speaker. 


\section{Introduction}

ATLAS Collaboration has a very rich $B$-physics programme. Using data collected during Run1, ATLAS observed two new states $\left(\chi_{b}(3 P)\right.$ and $\left.B_{c}^{ \pm}(2 S)\right)$ [1, 2], studied quarkonia production [3-10] and measured properties of various $b$-hadrons [11-15]. In these proceedings, a small selection of ATLAS results is presented.

The ATLAS detector [16] is a general purpose detector with cylindrical geometry ${ }^{1}$ and forwardbackward symmetric coverage in pseudorapidity $(\eta)$. The detector consists of inner tracking detectors (ID), calorimeters, the muon spectrometer (MS) and has a three-level trigger system. The ID directly surrounds the beam pipe and is immersed in a $2 \mathrm{~T}$ axial magnetic field generated by a superconducting solenoid.

\section{Study of $B_{c}^{+} \rightarrow J / \psi D_{s}^{+}$and $B_{c}^{+} \rightarrow J / \psi D_{s}^{*+}$ decays}

The $B_{c}^{+}$meson is the only weakly decaying particle consisting of two heavy quarks. Its decays to charmonium and a $D_{s}^{+}$or a $D_{s}^{*+}$ meson can occur via colour-suppressed and colour-favoured diagrams, as well as via the weak annihilation diagrams. This analysis uses the combination of $4.9 \mathrm{fb}^{-1}$ and $20.6 \mathrm{fb}^{-1}$ of $p p$ data at $\sqrt{s}=7 \mathrm{TeV}$ and $\sqrt{s}=8 \mathrm{TeV}$ respectively and presents the measurement of $B_{c}^{+} \rightarrow J / \psi D_{s}^{+}$and $B_{c}^{+} \rightarrow J / \psi D_{s}^{*+}$ decays (see figure 1 left), normalised to $B_{c}^{+} \rightarrow$ $J / \psi \pi^{+}$decays and the polarisation in $B_{c}^{+} \rightarrow J / \psi D_{s}^{*+}$ mode [14].
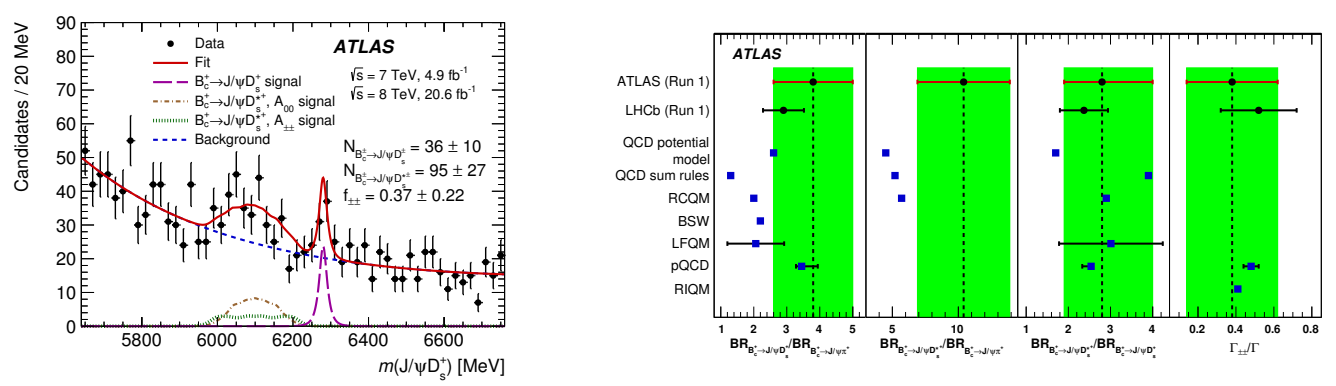

Figure 1: Left: Mass distribution of $J / \psi D_{s}^{+}$candidates. The contribution of the $B_{c}^{+} \rightarrow J / \psi D_{s}^{+}$decay is shown with the magenta long-dashed line. Brown dash-dot and green dotted lines show the $B_{c}^{+} \rightarrow J / \psi D_{s}^{*+}$ $A_{00}$ and $A_{ \pm \pm}$component contributions, respectively. Blue dashed line shows the background model. Right: Comparison of the results with other experiments and theoretical predictions [14].

The $D_{s}^{+} \rightarrow \phi(\rightarrow K K) \pi^{+}$mode is used for the reconstruction of the $D_{s}^{+}$meson, while the $D_{s}^{*+}$ decays into a $D_{s}^{+}$meson and a soft photon or $\pi^{0}$, where no attempt to reconstruct the soft neutral particles is attempted. The $B_{c}^{+} \rightarrow J / \psi D_{s}^{*+}$ decay is described by three helicity amplitudes

\footnotetext{
${ }^{1}$ ATLAS uses a right-handed coordinate system with its origin at the nominal interaction point (IP) in the centre of the detector and the $z$-axis along the beam pipe. The $x$-axis points from the IP to the centre of the LHC ring, and the $y$-axis points upward. Cylindrical coordinates $(r, \phi)$ are used in the transverse plane, $\phi$ being the azimuthal angle around the beam pipe. The pseudorapidity $\eta$ is defined in terms of the polar angle $\theta$ as $\eta=-\ln \tan (\theta / 2)$ and the transverse momentum $p_{\mathrm{T}}$ is defined as $p_{\mathrm{T}}=p \sin \theta$. The rapidity is defined as $y=0.5 \ln \left(\left(E+p_{z}\right) /\left(E-p_{z}\right)\right)$, where $E$ and $p_{z}$ refer to energy and longitudinal momentum, respectively. The $\eta-\phi$ distance between two particles is defined as $\Delta R=\sqrt{(\Delta \eta)^{2}+(\Delta \phi)^{2}}$.
} 
$\left(A_{++}, A_{--}\right.$and $\left.A_{00}\right)$ since it is a transition of a pseudoscalar meson into a pair of vector states. The $J / \psi$ meson is reconstructed in its di-muon decay mode.

As illustrated in figure 1 (right), the following ratios of branching fractions are measured and compared to experimental measurements and theoretical calculations: $\mathscr{R}_{D_{s}^{+} / \pi^{+}}=\mathscr{B}_{B_{c}^{+} \rightarrow J / \psi D_{s}^{+}} / \mathscr{B}_{B_{c}^{+} \rightarrow J / \psi \pi^{+}}=$ $3.8 \pm 1.1$ (stat.) \pm 0.4 (syst.) $\pm 0.2(\mathrm{BF}), \mathscr{R}_{D_{s}^{*+} / \pi^{+}}=\mathscr{B}_{B_{c}^{+} \rightarrow J / \psi D_{s}^{*+}} / \mathscr{B}_{B_{c}^{+} \rightarrow J / \psi \pi^{+}}=10.4 \pm 3.1$ (stat.) \pm 1.5 (syst.) $\pm 0.6(\mathrm{BF})$ and $\mathscr{R}_{D_{s}^{*+} / D_{s}^{+}}=\mathscr{B}_{B_{c}^{+} \rightarrow J / \psi D_{s}^{*+}} / \mathscr{B}_{B_{c}^{+} \rightarrow J / \psi D_{s}^{+}}=2.8_{-0.8}^{+1.2}$ (stat.) \pm 0.07 (syst.), where $\mathrm{BF}$ is the uncertainty from the $\mathscr{B}_{D_{s}^{+} \rightarrow \phi(K K) \pi^{+}}$knowledge. Finally, the relative contribution of the $A_{ \pm \pm}$component in $B_{c}^{+} \rightarrow J / \psi D_{s}^{*+}$ decay is measured to be $\Gamma_{ \pm \pm} / \Gamma=0.38 \pm 0.23$ (stat.) \pm 0.07 (syst.). All measurements of branching fraction ratios and the transverse polarisation fraction are found to be well described by theoretical approaches and other experimental measurements.

\section{Observation of $\Lambda_{b}$ in the decay $\Lambda_{b}^{0} \rightarrow \psi(2 S) \Lambda^{0}$}

ATLAS Collaboration observed the $\Lambda_{b}^{0} \rightarrow \psi(2 S) \Lambda^{0}$ decay [15] and measured the branching ratio of $\Lambda_{b}^{0} \rightarrow \psi(2 S) \Lambda^{0}$ (see figure 2 left) and $\Lambda_{b}^{0} \rightarrow J / \psi \Lambda^{0}$, which theoretical calculations predicts 0.8 with an uncertainty of about 0.1 . The $J / \psi$ and $\psi(2 S)$ mesons are reconstructed to their di-muon decay mode (see figure 2 middle) and the $\Lambda^{0} \rightarrow p \pi^{-}$(see figure 2 right).
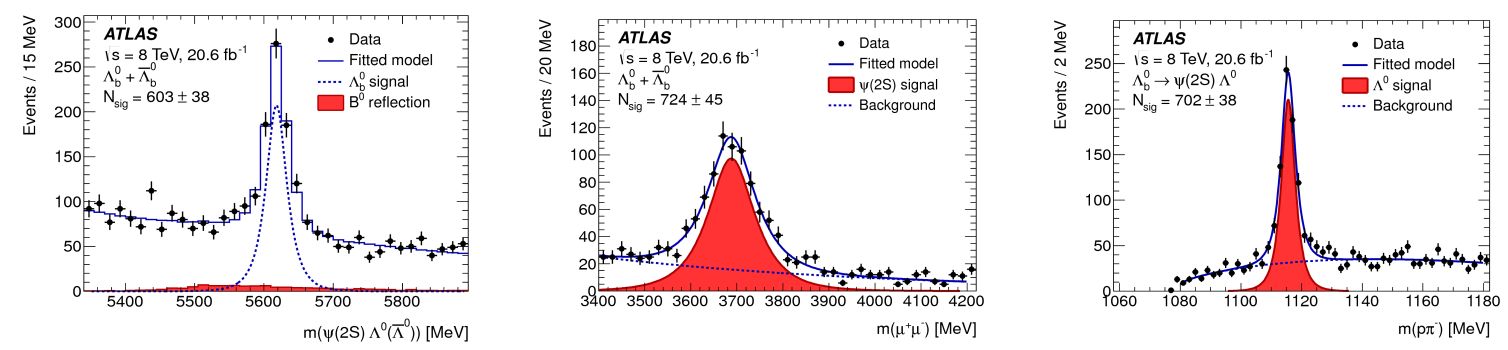

Figure 2: Left: Invariant mass distributions for the combined sample of the selected $\Lambda_{b}^{0}$ and anti- $\Lambda_{b}^{0}$ candidates obtained after their fits to the $\Lambda_{b}^{0} \rightarrow \psi(2 S) \Lambda^{0}$. Middle: The $m(\mu \mu)$ distributions for $\Lambda_{b}^{0} \rightarrow \psi(2 S) \Lambda^{0}$ candidates. Right: The $m(p \pi)$ distributions for $\Lambda_{b}^{0} \rightarrow \psi(2 S) \Lambda^{0}$ candidates [15].

The branching ratio of the two $\Lambda_{b}^{0} \rightarrow J / \psi \Lambda^{0}$ and $\Lambda_{b}^{0} \rightarrow \psi(2 S) \Lambda^{0}$ is calculated as $\Gamma\left(\Lambda_{b}^{0} \rightarrow\right.$ $\left.\psi(2 S) \Lambda^{0}\right) / \Gamma\left(\Lambda_{b}^{0} \rightarrow J / \psi \Lambda^{0}\right)=N_{\text {cor }}\left(\Lambda_{b}^{0} \rightarrow \psi(2 S) \Lambda^{0}\right) / N_{\text {cor }}\left(\Lambda_{b}^{0} \rightarrow J / \psi \Lambda^{0}\right) \cdot \mathscr{B}(J / \psi \rightarrow \ell \ell) / \mathscr{B}(\psi(2 S) \rightarrow$ $\ell \ell$ ), where $\mathscr{B}$ is the branching fraction of the corresponding charmonium decay to a lepton pair and $N_{\text {cor }}$ is the number of $\Lambda_{b}^{0}$ signal events in the two decay modes, after correcting for detector effects and selection efficiencies.

Using $20.6 \mathrm{fb}^{-1}$ of $p p$ data at $\sqrt{s}=8 \mathrm{TeV}$, the measured branching ratio of the two $\Lambda_{b}^{0}$ decays is found to be $\Gamma\left(\Lambda_{b}^{0} \rightarrow \psi(2 S) \Lambda^{0}\right) / \Gamma\left(\Lambda_{b}^{0} \rightarrow J / \psi \Lambda^{0}\right)=0.501 \pm 0.033$ (stat) \pm 0.016 (syst) $\pm 0.011(\mathscr{B})$. The measured branching ratio falls inside the range of analogous $B$ meson decays $(0.5-0.8)$.

\section{Prompt and non-prompt $J / \psi$ and $\psi(2 S)$ production at $\sqrt{s}=2.76,7,8$ and $13 \mathrm{TeV}$}

Measurements of quarkonia (heavy $q \bar{q}$ bound states) production processes is a powerful tool to study QCD. Although more than forty years passed since the discovery of the $J / \psi$ meson, hidden flavour production in hadronic collisions still provides challenges to both theory and experiment. ATLAS Collaboration used $p p$ collision data in a variety of centre-of-mass energies (2.76 TeV [3], 
7 and $8 \mathrm{TeV}$ [4] and $13 \mathrm{TeV}$ [5]) to accurately measure the production of quarkonia in a broad range of transverse momentum and rapidity.

ATLAS measurements include production cross-sections (see figure 3 left and middle), nonprompt production fractions (see figure 3 right) and production ratios of $\psi(2 S)$ to $J / \psi$ mesons. Measurements have been compared to theoretical predictions, where a reasonable agreement is observed. A consistency is also found by comparing ATLAS results to previous experimental measurements in overlapping phase spaces. Studying the dependence of the non-prompt production fraction from the $\sqrt{s}$, using energies from $\sqrt{s}=1.96 \mathrm{TeV}$ to $\sqrt{s}=13 \mathrm{TeV}$, a significant difference between the $\sqrt{s}=7 \mathrm{TeV}$ measurement and measurements at lower energies is observed (see 3 right).
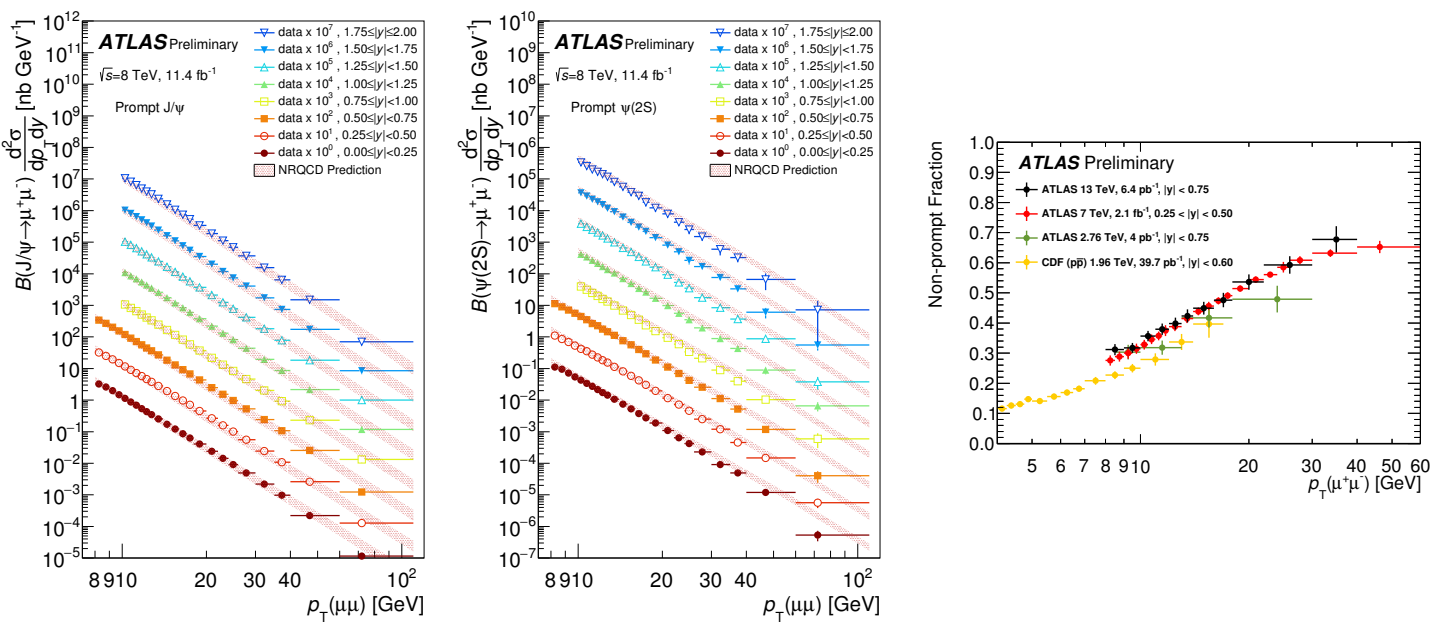

Figure 3: Differential prompt cross-section times dimuon branching fraction of $J / \psi($ left) and $\psi(2 S)$ (middle) as a function of $p_{\mathrm{T}}$ for each slice of rapidity. For each increasing rapidity slice, an additional scaling factor of 10 is applied to the plotted points for visual clarity. Along with the measurements the NLO NRQCD theory predictions are presented. Right: Non-prompt differential $J / \psi$ production fraction measured in the most central rapidity region $(|y|<0.75)$ compared to previous measurements from ATLAS in $p p$ collisions at 2.76 and $7 \mathrm{TeV}$, and from $\mathrm{CDF}$ in $p \bar{p}$ collisions at $\sqrt{s}=1.96 \mathrm{TeV}[4,5]$.

\section{Associated production of $Z$ bosons with prompt and non-prompt $J / \psi$ mesons}

Studying the associated production of electroweak bosons with quarkonia can provide constraints on the contributions from colour-octet and colour-singlet processes and can also be used for the understanding of heavy flavour production with vector bosons. Additionally, it is a powerful tool to model single and double parton interactions in $p p$ collisions. Using $20.3 \mathrm{fb}^{-1}$ of $p p$ collision data at $\sqrt{s}=8 \mathrm{TeV}$, ATLAS Collaboration observed the production of prompt and non-prompt $J / \psi(\rightarrow \mu \mu)$ mesons in association with a $Z(\rightarrow \ell \ell)$ boson $(\ell=\mu, e)$ with a $5 \sigma$ and $9 \sigma$ significance respectively [10].

Measurements include the fiducial cross-section ratio, defined as $R_{Z+J / \psi}^{\mathrm{fid}}=\sum_{p_{\mathrm{T}} \text { bins }}\left[N^{\mathrm{ec}}(Z+\right.$ $\left.J / \psi)-N_{\text {pileup }}^{\text {ec }}\right] / N(Z)$ which is measured to be $(36.8 \pm 6.7$ (stat. $) \pm 2.5$ (syst. $\left.)\right) \times 10^{-7}$ for the prompt and $(65.8 \pm 9.2$ (stat.) \pm 4.2 (syst. $)) \times 10^{-7}$ for the non-prompt $J / \psi+Z$ production, inclusive crosssection ratio (total and as a function of $p_{\mathrm{T}}^{J / \psi}$ as shown in figure 4), assuming unpolarised $J / \psi$ de- 
cays, which is measured to be $(63 \pm 13$ (stat.) \pm 5 (syst. $) \pm 10$ (pol. $)) \times 10^{-7}$ and $(102 \pm 15$ (stat. $) \pm$ 5 (syst. $) \pm 3$ (pol. $)) \times 10^{-7}$ for prompt and non-prompt $J / \psi$ production respectively. Finally, subtracting DPS contributions ( $Z$ and $J / \psi$ produced from two different pairs of interacting partons), in order to compare the SPS ( $Z$ and $J / \psi$ produced from one pair of interacting partons) measurement with theoretical models, the $Z+$ prompt $J / \psi$ production cross-section ratio was found to be $(45 \pm 13$ (stat.) \pm 6 (syst.) \pm 10 (pol. $)) \times 10^{-7}$ and $Z+$ non-prompt $J / \psi(94 \pm 15$ (stat. $) \pm 5$ (syst. $) \pm$ $3($ pol. $)) \times 10^{-7}$.

The $Z$ boson and the $J / \psi$ meson can be produced either from SPS or DPS processes. DPS events are expected to be distributed uniformly along the azimuthal angle between the $Z$ boson and the $J / \psi(\Delta \phi)$ because the $Z$ and $J / \psi$ particles are produced from two independent scatters. SPS events are expected to show a back-to-back correlation $\Delta \phi=\pi$, since they originate from a single parton interaction. Using the kinematic properties of the two final state particles and the assumption that the first bin in the $\Delta \phi(Z, J / \psi)<\pi / 5$ distribution is DPS dominated, the data uncertainties and uncertainties inherent in the DPS estimate allow a lower limit $\sigma_{\text {eff }}>5.3 \mathrm{mb}(3.7 \mathrm{mb})$ at $68 \%(95 \%)$ confidence level to be extracted.
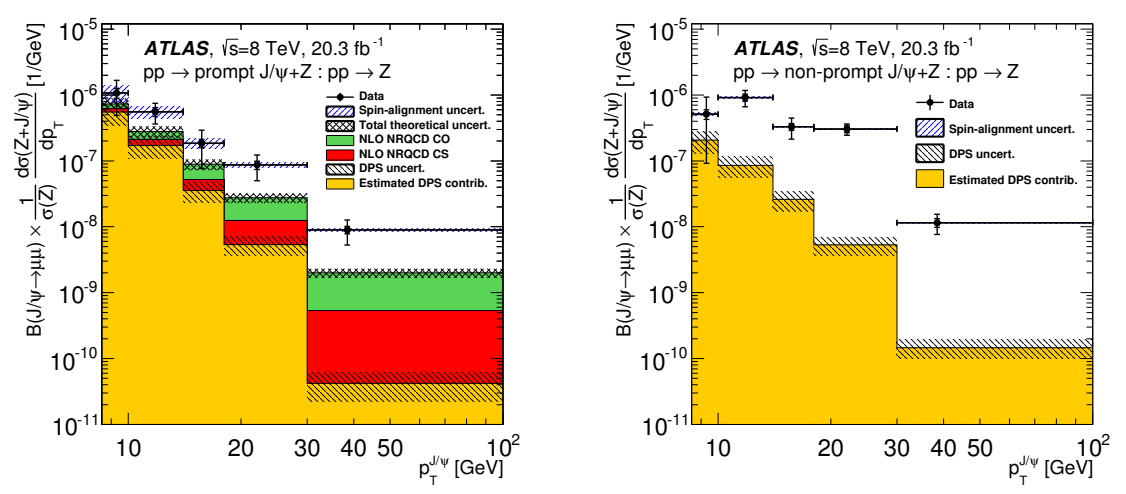

Figure 4: Production cross-section of prompt (left) and (non-promtpt) $J / \psi$ mesons in association with a $Z$ boson as a function of $p_{\mathrm{T}}^{J / \psi}$, normalised to the inclusive $Z$ cross-section. Overlaid on the prompt measurement is the contribution to the total signal originating from DPS interactions as well as theoretical predictions at NLO accuracy for the SPS contributions from CS and CO processes (which are added to the DPS estimate) and presented in comparison to the data as solid bands [10].

\section{Conclusions}

ATLAS Collaboration used Run1 data to measure with great precision and observe new processes in the heavy flavour sector. Already with the very first $\mathrm{pb}^{-1}$ of $\sqrt{s}=13 \mathrm{TeV}$ of data, observed the difference in $J / \psi$ non-prompt fraction centre-of-mass energy dependence, promising a fruitful Run2 period.

\section{Acknowledgments}

The present work was co-funded by the European Union (European Social Fund ESF) and Greek national funds through the Operational Program "Education and Lifelong Learning" of the National Strategic Reference Framework (NSRF) 2007-2013. ARISTEIA-1893-ATLAS MICROMEGAS. 


\section{References}

[1] ATLAS Collaboration, Observation of a new $\chi_{b}$ state in radiative transitions to $\Upsilon(1 S)$ and $\Upsilon(2 S)$ at ATLAS, Phys. Rev. Lett. 108, 152001 (2012), arXiv:1112.5154 [hep-ex] .

[2] ATLAS Collaboration, Observation of an Excited $B_{c}^{ \pm}$Meson State with the ATLAS Detector, Phys. Rev. Lett. 113, 212004 (2014), arXiv:1407.1032 [hep-ex] .

[3] ATLAS Collaboration, Study of $J / \psi$ and $\psi(2 \mathrm{~S})$ production in $\sqrt{s_{\mathrm{NN}}}=5.02 \mathrm{TeV} p+\mathrm{Pb}$ and $\sqrt{s}=2.76 \mathrm{TeV} p p$ collisions with the ATLAS detector, ATLAS-CONF-2015-023, (2015).

[4] ATLAS Collaboration, Measurement of the differential cross-sections of prompt and non-prompt production of $J / \psi$ and $\psi(2 S)$ in $p p$ collisions at $\sqrt{s}=7$ and $8 \mathrm{TeV}$ with the ATLAS detector, ATLAS-CONF-2015-024, (2015).

[5] ATLAS Collaboration, Measurement of the differential non-prompt $J / \psi$ production fraction in $\sqrt{s}=13 \mathrm{TeV} p p$ collisions at the ATLAS experiment, ATLAS-CONF-2015-030, (2015).

[6] ATLAS Collaboration, Measurement of the production cross-section of $\psi(2 S) \rightarrow J / \psi\left(\rightarrow \mu^{+} \mu^{-}\right) \pi^{+} \pi^{-}$in pp collisions at $\sqrt{s}=7$ TeV at ATLAS, JHEP 09, 79 (2014), arXiv:1407.5532 [hep-ex].

[7] ATLAS Collaboration, Measurement of $\chi_{c 1}$ and $\chi_{c 2}$ production with $\sqrt{s}=7 \mathrm{TeV} p p$ collisions at ATLAS, JHEP 07, 154 (2014), arXiv:1404.7035 [hep-ex] .

[8] ATLAS Collaboration, Measurement of Upsilon production in $7 \mathrm{TeV}$ pp collisions at ATLAS, Phys. Rev. D87, 052004 (2013), arXiv:1211.7255 [hep-ex].

[9] ATLAS Collaboration, Measurement of the production cross section of prompt $J / \psi$ mesons in association with a $W^{ \pm}$boson in $p p$ collisions at $\sqrt{s}=7 \mathrm{TeV}$ with the ATLAS detector, JHEP 04, 172 (2014), arXiv:1401.2831 [hep-ex].

[10] ATLAS Collaboration, Observation and measurements of the production of prompt and non-prompt $J / \psi$ mesons in association with a $Z$ boson in $p p$ collisions at $\sqrt{s}=8 \mathrm{TeV}$ with the ATLAS detector, Eur. Phys. J. C75, 229 (2015), arXiv:1412.6428 [hep-ex].

[11] ATLAS Collaboration, Measurement of the parity-violating asymmetry parameter $\alpha_{b}$ and the helicity amplitudes for the decay $\Lambda_{b}^{0} \rightarrow J / \psi+\Lambda^{0}$ with the ATLAS detector, Phys. Rev. D89, 092009 (2014), arXiv:1404.1071 [hep-ex].

[12] ATLAS Collaboration, Measurement of the differential cross-section of $B^{+}$meson production in pp collisions at $\sqrt{s}=7 \mathrm{TeV}$ at ATLAS, JHEP 10,042 (2013), arXiv:1307.0126 [hep-ex] .

[13] ATLAS Collaboration, Measurement of the $\Lambda_{b}^{0}$ lifetime and mass in the ATLAS experiment, Phys. Rev. D87, 032002 (2013), arXiv:1207.2284 [hep-ex].

[14] ATLAS Collaboration, Study of the $B_{c}^{+} \rightarrow J / \psi D_{s}^{+}$and $B_{c}^{+} \rightarrow J / \psi D_{s}^{*+}$ decays with the ATLAS detector, arXiv:1507.07099 [hep-ex].

[15] ATLAS Collaboration, Measurement of the branching ratio $\Gamma\left(\Lambda_{b}^{0} \rightarrow \psi(2 S) \Lambda^{0}\right) / \Gamma\left(\Lambda_{b}^{0} \rightarrow J / \psi \Lambda^{0}\right)$ with the ATLAS detector, arXiv:1507.08202 [hep-ex].

[16] ATLAS Collaboration, The ATLAS Experiment at the CERN Large Hadron Collider, JINST 3, S08003 (2008). 